\title{
Effect of Wound Dressing with Fresh Kiwifruit on healing of Cutaneous Wound in Rats
}

\author{
Iran Goudarzi ${ }^{1,}$; Taghi Lashkarbolouki ${ }^{1}$; Mahdi Khorshidi ${ }^{1}$; Mohammad Taghi Ghorbanian ${ }^{1}$ \\ ${ }^{1}$ Faculty of Biology, Institute of Biological Sciences, Damghan University, Damghan, Iran \\ *Corresponding author: Iran Goudarzi, Faculty of Biology, Institute of Biological Sciences, Damghan University, Damghan, Iran., E-mail: irangoudarzi@du.ac.ir
}

Received: January 27, 2014; Accepted: February 2, 2014

\begin{abstract}
Background: The kiwi fruit is known to have dramatic antibacterial, debridement, wound contracture, and angiogenic effects. We propose that kiwifruit is an ideal candidate to enhance the process of wound healing. The present study assessed the effects of wound kiwifruit dressing on cutaneous wound healing in rat.

Materials and Methods: In this experimental study, 30 male Wistar rats were randomly divided into 2 groups of control and kiwifruit group. A full-thickness dermal incision (35mm length) was made on the right side of the paravertebral region. In the control group, one day after wound induction wounds was dressed with Vaseline sterile gauze after normal saline irrigation. In the second group, the wounds were dressed with kiwifruit. Wound healing was evaluated by measuring surface area, percentage of healing, duration of healing, and wound tensile strength.

Results: Obtained results showed that the duration of wound healing in kiwi group in comparison with the control was significantly decreased. The amount of wound healing in percent was also significantly different between control and kiwi groups at days 3,6 ( $p<0.001), 9$ ( $p<0.05), 12$ and 14 ( $p<0.01$ ). Comparisons of wound length between control and kiwi group per day showed that kiwi group had significantly lower wound length on day $9,12,14$ and 16 ( $p<0.01,0.001,0.01$ and 0.01 , respectively) in comparisons to control group. Also, the wound tensile strength in kiwi group also was significantly greater than the control animals $(\mathrm{p}<0.01)$.

Conclusion: We concluded that our study provides some evidence to support the use of kiwi to accelerate wound healing.
\end{abstract}

Keywords:Cutaneous wound; Wound healing; Kiwifruit; Rat

\section{Introduction}

Wound healing is a dynamic process involving cellular, molecular, biochemical, and physiological phenomena that result in connective tissue repair and fibrous scar formation and lead to the restoration of the anatomical continuity and functional status of the skin [1].

Healing of wound is a specific biological process related to the general phenomenon of growth and tissue regeneration. There are several reports describing the various biological and physiological stages of healing. The wound healing process can be summarized into five independent and overlapping stages, including hemostasis, inflammation, migration, proliferation and maturation $[2,3]$.

All these phases are strictly connected and play a key role for a complete and proper restoration of the injured tissue. There are many factors that can affect wound healing by interfering with one or more phases in this process, thus causing improper or impaired tissue repair. Research on wound healing drugs is a developing area in modern biomedical sciences and the search for compounds derived from plants constitutes a significant part of such studies. Although many natural products have been claimed to have healing effects, most do not have well-controlled scientific data. They need to be characterized in respect to the active chemical compounds, elucidation of the molecular mechanisms of their actions, demonstration of the real efficacy by in vivo studies and, finally, demonstration of their safety and effectiveness in clinical trials [4].

The kiwifruit originated $>700$ years ago in China. It was later introduced in New Zealand and California, where the first major planting occurred in 1960. Some clinical effects of kiwifruit ingredients such as ascorbic acid (as a scavenger), antibacterial agents, and actinidin (a potent protein-dissolving enzyme) have been reported in the literature [5-7]. The proteolytic activities of actinidin and papain (proteolytic enzyme in papaya) are well-known commercially for meat tenderizing. Cheap and widely available in Africa, topical papaya is effective in desloughing wounds and limiting burn wound infection and is currently used in some regions of Africa. Previous study has shown the dramatic effect of kiwifruit dressing on deep second-degree burn wound healing. Debridement of devitalized tissue with no deleterious effects on the healing process keeps the wound surface clean and humid and prevents bacterial proliferation. 
They suggested that the dramatic antibacterial, debridement, wound contracture, and angiogenic effect of kiwifruit induced a significant wound healing in burn ulcers and might be useful in treating chronic ulcers, such as bed sores [8]. Also, Hafezi et al. studied effect of kiwifruit on standard 3rd degree burn wound healing. They reported that debridement, neo-epithelialization and scar contraction were faster in the kiwi-treated group than in the untreated group. Following rapid enzymatic debridement, healing appeared to progress normally, with no evidence of damage to the adjacent healthy tissue [9].

Regarding to, kiwifruit contains antibacterial, scavenger agents and proteolytic enzymes, which may improve the wound healing and since using natural compounds has lower side effects comparing to chemical substances, the effect of kiwifruit dressing on the healing process of cutanous wound in male rats were examined in this study.

\section{Materials and Methods}

In this experimental study, 30 male Wistar rats, weighting 180-220 g, from Pasteur institute were used in this study. Rats were kept in standard cages, and fed ad libitum. The room temperature was also kept constant at $21 \pm 0.5^{\circ} \mathrm{C}$. The animals were weighed at beginning of the experiment and then weekly throughout the experiment. All animals were under care and used in the experiments according to the guidelines of the ethical committee of Damghan University.

Rats were randomly divided into 2 control and kiwifruit treated groups. Animals were anaesthetized with an intraperitoneal injection of $10 \mathrm{mg} / \mathrm{kg}$ Rampan (Bayer, Leverkusen, Germany) and $100 \mathrm{mg} / \mathrm{kg}$ ketamine hydrochloride (Gedeon Richter, Budapest, Hungary). After shaving and cleaning, a full-thickness incision $(35 \mathrm{~mm}$ in length) was made parallel to and at a distance of $1.5 \mathrm{~cm}$ on the right of the dorsal midline for each animal (Figure 1).

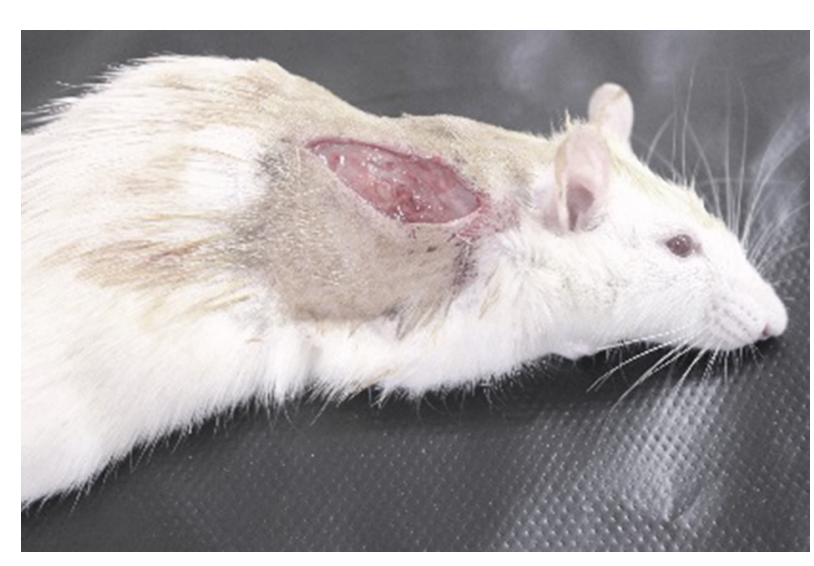

Figure 1. A full-thickness wound was excised parallel to and at a distance of $1.5 \mathrm{~cm}$ on right of the dorsal midline for each animal
Postsurgically, the rats were returned to their cages and housed individually in order to avoid cannibalistic behavior. In the control group, one day after wound induction wounds was dressed with vaseline sterile gauze after normal saline irrigation. In the second group, the wounds were dressed with kiwifruit. The dressings were changed each day in 2 groups.

To evaluate wound healing, some parameters such as duration of healing, wound length, surface area of wound, and tensile strength were used. For surface area measurement, rats were anesthetized with ether inhalation, and a trace of each wound surface was made with tracing paper affixed to a millimeter paper, and squares located inside the trace were counted. The surface area was measured on zero (surgery day), 3, 6, 9, 12, 14, 16, 18 days after surgery. Therefore, this measurement was carried out until full healing occurred. The healing percentage was calculated by dividing the maximum surface area or of the wounds by that measured on 3, 6, 9, 12, 14, 16,18 days after surgery. The duration of wound healing was the time taken for full contraction of wound [10].

Twenty-one days after the surgery, animals were killed by an overdose of ether and the tensile strength of each scar was measured. A rectangular segment of the skin $(5 \pm 1 \mathrm{~cm})$, perpendicular to the incision, was removed and a $1 \mathrm{~cm}$ width from the center of incision was taken (to ensure the exclusion of unincised skin of the removed segment). The subcutaneous tissue was carefully removed from each skin sample, moistened with a saline solution $(0.9 \% \mathrm{NaCl})$ and immediately taken to the tensiometer skin sample was connected to a bag of water by a clip at one end and to the tensiometer by a second clip at the other end. Water added to the bag at a rate of $2 \mathrm{~g} / \mathrm{sec}$ ond (regulated by a value) until complete rupture of the wound. The weight of the bag and water was then measured and noted as the tensile strength [11].

The results were expressed as Mean \pm SEM. Data were analyzed through independent student t-test, using SPSS16 and a statistical p value less than 0.05 was considered significant.

\section{Results}

Figure 2 shows that duration of healing time in kiwi treated animals is significantly $(\mathrm{p}<0.001$, student $\mathrm{t}$-test) shorter than control group. Therefore, there was faster of wound healing in kiwi treated animals. Woundhealing percentage was calculated using the equation (S2-S1)/ S2×100, where S2 and S1 are wound surface area on the surgery day, and days on 3, 6, 9, 12, 14, 16, 18 days, respectively.

The area of wound was significantly different at 3rd $(\mathrm{p}<0.001)$, sixth $(\mathrm{p}<0.001)$, ninth $(\mathrm{p}<0.05)$, twelfth $(\mathrm{p}<0.01)$ and fourteenth $(\mathrm{p}<0.01)$ days between control and kiwi treated groups. Comparing the percentage of healing on different days showed an increased rate of healing in kiwi treated animals (Figure 3). 


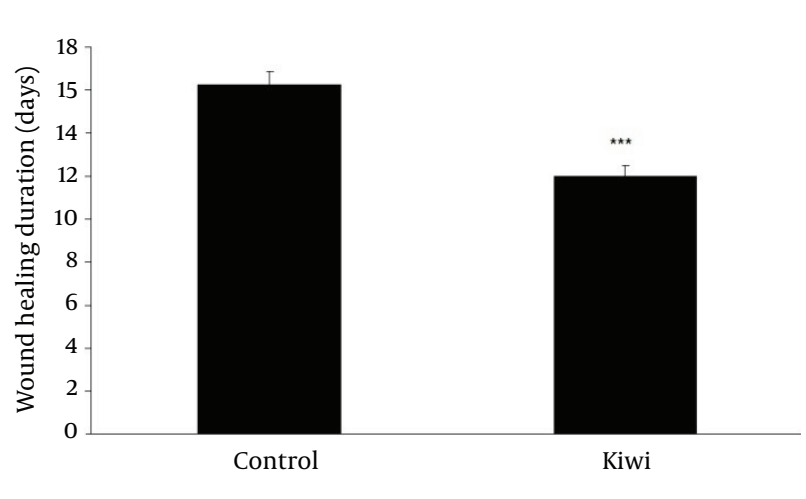

Figure 2. Comparison of healing time between control and kiwi treated rats. There was significant decrease in wound-healing time in kiwi treated rats compared to control group. Values are mean \pm SEM, $\mathrm{N}=14$ for each group. ${ }^{* * *} \mathrm{p}<0.001$.

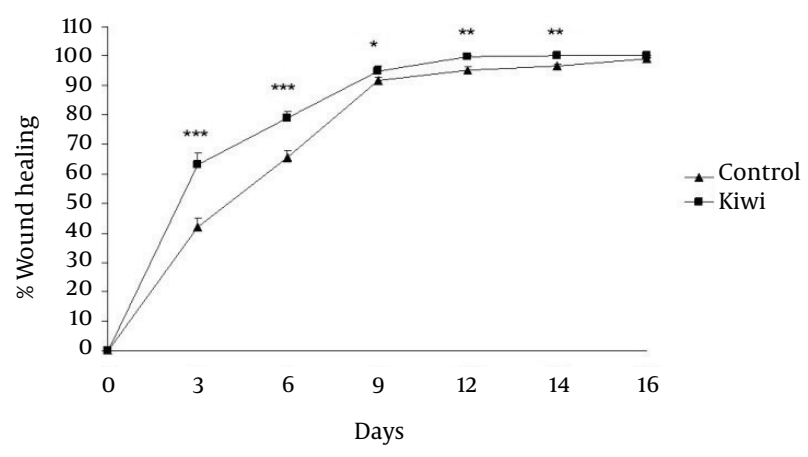

Figure 3. Effect of kiwifruit dressing of wound on wound-healing percentage in rats. The percentage of wound healing showed significantly increase on day3, day 6, day 9, day 12 and day 14 in kiwi treated rats as compared with control group. Values are mean \pm SEM, $\mathrm{N}=15$ for each group. ${ }^{*} \mathrm{p}<0.05,{ }^{* *} \mathrm{p}<0.01,{ }^{* * *} \mathrm{p}<0.001$.

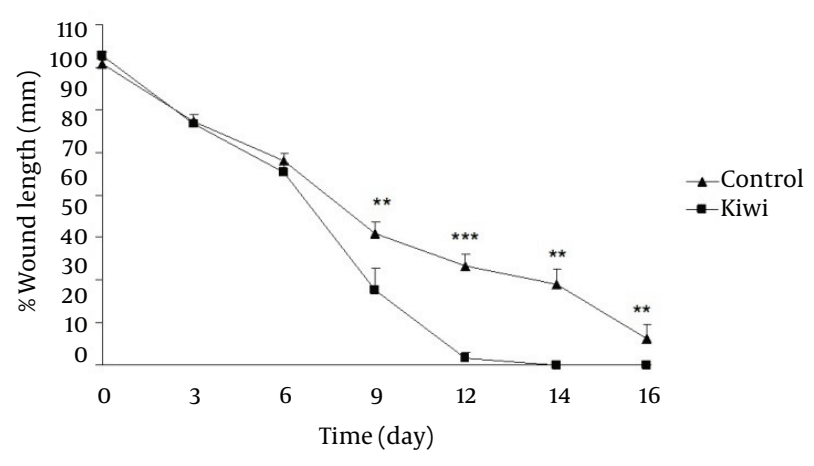

Figure 4. The effect of kiwifruit dressing of wound on wound length per day. Comparisons of wound length between control and kiwi treated rats per day showed that kiwi treated group had significantly lower wound length on day 6, 9, 12 and 14 in comparisons to control group. ${ }^{* *} \mathrm{p}<0.01$, ${ }^{* * *} \mathrm{p}<0.001$.

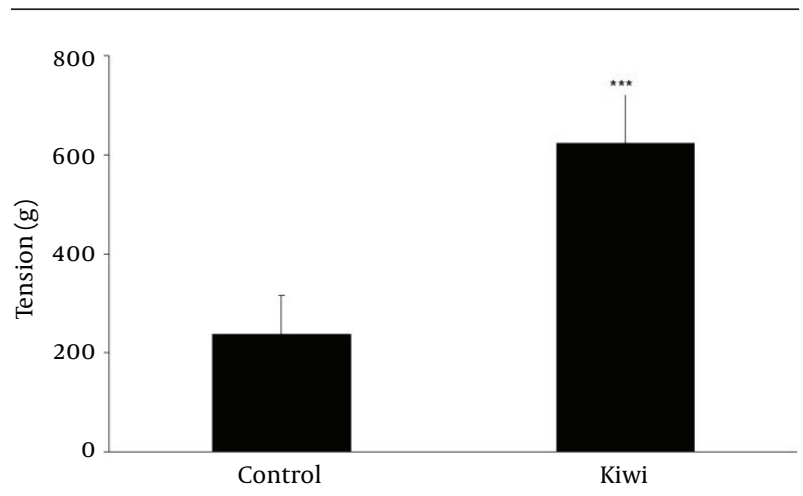

Figure 5. Effect of kiwi fruit dressing of wound on the tensile strength of scar tissue 21 days after surgery. The kiwi fruit significantly enhanced the tensile strength of scar tissue than control group. Values are mean $\pm S E M$, $\mathrm{N}=6$ for each group. ${ }^{* * *} \mathrm{p}<0.001$.

Figure 4 shows the wound length $(\mathrm{mm})$ in different days during wound healing. There was significant reduction of wound length on day $9(\mathrm{p}<0.01)$, day $12(\mathrm{p}<0.001)$, day $14(\mathrm{p}<0.01)$ and day $14(\mathrm{p}<0.01)$ in kiwi treated group in comparisons to control group. Next, 21 days after wounding, the effect of kiwifruit dressing on tensile strength of each scar was investigated. Figure 5 shows the effect of kiwifruit dressing on tensile strength scar tissue in two groups. As seen, the tensile strength of kiwi treated group is much higher than control groups (Figure 5, $\mathrm{p}<0.001$ ).

\section{Discussion}

The local effect of Kiwi fruit on healing of cutaneous wound in male rats was examined in this study. The findings of the present study showed a significant decrease in wound healing duration, increase of wound healing percentage and tensile strength in kiwi treated rats than control group. These observations probably indicate the healing effect of kiwifruit on rats' cutaneous wound.

Wounds are common clinical entities in day-to-day life, which may be major or minor. The process of wound healing can be classified into 5 phases-cellular phase (granulation), narrowing of wound area (wound contraction), collagen deposition (collagenation), epithelial covering (epithelialisation) and scar remodeling (cicatrisation). These phases are concurrent but independent of each other. Any agent that accelerates the process is a promoter of wound healing [12]. Accordingly, the healing effect of kiwifruit is probably through the advancement of some of these steps.

Papaya is widely used in hot climate countries as traditional remedy in wound treatment. Its therapeutical value has been proven in several animal and human trials [13-15]. Papain and chymopapain are known to be effective in desloughing necrotic tissue, prevention of infection and the antimicrobial and antioxidant properties related to hydroxyl scavenging and iron chelating properties [15]. Moreover, they decrease the risk of oxidative damage 
to tissues. The antibacterial and desloughing effect of papaya is related to ingredients such as antibacterial agents and proteolytic enzymes (papain, leukopapain, chymopapain). Comparably, kiwifruit contains antibacterial and scavenger agents in addition to proteolytic enzymes, which may improve the wound healing [8]. In this study, better wound contraction may result not only from the early and full epithelialization, but also from debridement effect of this fruit.

Basile et al. demonstrated significant antibacterial activity on animals treated with kiwifruit dressing in comparison with those who received silver sulfadiazine cream [7]. Mohajeri et al. indicated that dressing with kiwifruit accelerate wound healing in burn ulcers and they suggested this effects is due to the dramatic antibacterial, debridement, wound contracture, and angiogenic effects of kiwifruit [8].

In wound healing, the strength and integrity of tissue is ensured by collagen accumulation and it's cross-linking.

The collagen chain can only be formed if proline and lysine are hydroxylated in ribosomes. In this synthesis, ascorbic acid is used as a cofactor in converting proline residues into hydroxyproline [16]. Consequently, ascrobate stimulates procollagen secretion and ascorbic acid deficiency impairs collagen synthesis [17].

Ascorbic acid has been reported to increase collagen deposition and tensile strength in experimental models of wound repair. Ascorbic acid is a very water-soluble compound which reacts with free radicals that arise in the aqueous compartments of tissues [18]. Fruits and vegetables are the major sources of ascorbic acid for the human system, which is unable to synthesize it but must secure it through dietary uptake [19]. Kiwifruit (Actinidia), one of the most popular fruits, is highly regarded for its high ascorbic acid content [20]. In our study, enhancement of tensile strength in kiwi treated wound probably is due to high ascorbic acid content of kiwi fruit. These results obtained from this study provide some evidence to support the use of kiwi to accelerate wound healing. The application of kiwi fruit and the similar healing fruits in treating the lesion leads to the results improvement, lower costs and lower antibiotic use in the patients. Further research is needed to determine the kiwi effect mechanisms in acceleration of wound healing in male rats.

\section{Acknowledgements}

The authors would like to thank the Deputy of Research of Damghan University for the financial supports that made this project possible (Code No. 91/Bio/109/204).

\section{Authors' Contributions}

All authors had equal role in design, work, statistical analysis and manuscript writing.

\section{Conflict of Interest}

The authors declare no conflict of interest.

\section{Funding/Support}

Institute of Biological Sciences of Damghan University.

\section{References}

1. Bowler PG. Wound pathophysiology, infection and therapeutic options. Ann Med. 2002;34(6):419-27.

2. Boateng JS, Matthews KH, Stevens HN, Eccleston GM. Wound healing dressings and drug delivery systems: a review. J Pharm Sci. 2008;97(8):2892-923.

3. Vasconcelos A, Cavaco-Paulo A. Wound dressings for a proteolytic-rich environment. Appl Microbiol Biotechnol. 2011; 90(2):445-60.

4. Majewska I, Gendaszewska-Darmach E. Proangiogenic activity of plant extracts in accelerating wound healing - a new face of old phytomedicines. Acta Biochim Pol. 2011;58(4):449-60.

5. Low C. The efficacy of fruit juices in disimpacting meat bolus obstruction. Otolaryngol Head Neck Surg. 2004;131(2):P166.

6. Motohashi N, Shirataki Y, Kawase M, Tani S, Sakagami H, Satoh K, et al. Cancer prevention and therapy with kiwifruit in Chinese folklore medicine: a study of kiwifruit extracts.J Ethnopharmacol. 2002;81(3):357-64.

7. Basile A, Vuotto ML, Violante U, Sorbo S, Martone G, CastaldoCobianchi R. Antibacterial activity in Actinidia chinensis, Feijoa sellowiana and Aberia caffra. International Journal of Antimicrobial Agents. 1997;8(3):199-203.

8. Mohajeri G, Masoudpour H, Heidarpour M, Khademi EF, Ghafghazi S, Adibi S, et al. The effect of dressing with fresh kiwifruit on burn wound healing. Surgery. 2010;148(5):963-8.

9. Hafezi F, Rad HE, Naghibzadeh B, Nouhi A, Naghibzadeh G. Actinidia deliciosa (kiwifruit), a new drug for enzymatic debridement of acute burn wounds. Burns. 2010;36(3):352-5.

10. Goudarzi I, Hajizadeh S, Salmani ME, Abrari K. Pulsed electromagnetic fields accelerate wound healing in the skin of diabetic rats. Bioelectromagnetics. 2010;31(4):318-23.

11. Bouzarjomehri FA, Sharafi AA, Firouzabadi SM, Hajizadeh S. Effects of low-frequency pulsed electromagnetic fields on wound healing in rat skin. Arch Iran Med. 2000;3(1):23-7.

12. Smith R. Recovery and tissue repair. Br Med Bull.1985;41(3):295-301.

13. Mikhal'chik EV, Ivanova AV, Anurov MV, Titkova SM, Pen'kov LY, Kharaeva ZF, et al. Wound-healing effect of papaya-based preparation in experimental thermal trauma. Bull Exp Biol Med. 2004;137(6):560-2.

14. Nayak SB, Pinto Pereira L, Maharaj D. Wound healing activity of Carica papaya L. in experimentally induced diabetic rats. Indian J Exp Biol. 2007;45(8):739-43.

15. Anuar NS, Zahari SS, Taib IA, Rahman MT. Effect of green and ripe Carica papaya epicarp extracts on wound healing and during pregnancy. Food Chem Toxicol. 2008;46(7):2384-9.

16. Ravindranath N, Little-Ihrig L, Phillips HS, Ferrara N, Zeleznik AJ. Vascular endothelial growth factor messenger ribonucleic acid expression in the primate ovary. Endocrinology. 1992; 131(1):254-60.

17. Peterkofsky B. Ascorbate requirement for hydroxylation and secretion of procollagen: relationship to inhibition of collagen synthesis in scurvy. Am J Clin Nutr. 1991;54(6 Suppl):1135S-40S.

18. Babior BM. Superoxide: a two-edged sword. Braz J Med Biol Res. 1997;30(2):141-55.

19. Li Y, Schellhorn HE. New developments and novel therapeutic perspectives for vitamin C. J Nutr. 2007;137(10):2171-84 .

20. Li M, Ma F, Liang D, Li J, Wang Y. Ascorbate biosynthesis during early fruit development is the main reason for its accumulation in kiwi. PLoS One. 2010;5(12). 